\section{Viele Ärzte decken inkompetente Kollegen}

Wenn es darum geht, Kollegen zu melden, die aus irgendwelchen Gründen nicht in der Lage sind, ihren Beruf korrekt auszuüben, sind viele Ärzte allzu zurückhaltend. Zu diesem Ergebnis kommt eine Umfrage unter rund 3000 Ärzten verschiedener Fachrichtungen in den USA. Während die meisten Ärzte grundsätzlich überzeugt waren, dass es sinnvoll sei, berufsunfähige Kollegen zu melden, gab immerhin jeder dritte Befragte an, auf die konkrete Situation nicht vorbereitet zu sein. $17 \%$ berichteten, dass sie in den letz- ten drei Jahren schon einmal Kontakt zu einem Kollegen hatten, an dessen Kompetenz sie zweifelten, aber nur zwei Drittel hatten dies an die zuständigen Stellen gemeldet. Klinikärzte waren eher dazu bereit als Niedergelassene. 19\% nannten als Grund für ihre Zurückhaltung, dass sie hofften, andere Kollegen würden das Problem für sie lösen, $15 \%$ meinten, dass eine Meldung ohnehin keine Konsequenzen haben würde, und $12 \%$ befürchteten Nachteile für sich selbst.

JAMA 2010;304:1871-93

\section{ALKOHOLKONSUM UND SCHLAGANFALLRISIKO \\ Keine Happy Hour}

Ein einziges alkoholisches Getränk scheint zu genügen: In der ersten Stunde nach dem Konsum verdoppelt sich - vorübergehend - das Schlaganfallrisiko. Das gilt nicht nur bei hochprozentigen Spirituosen, sondern auch bei Wein oder Bier. Darauf weisen die Resultate einer Studie hin, in der 390 Patienten mit ischämischem Schlaganfall zu den Stunden vor dem Ereignis befragt wurden.

Hoher Alkoholkonsum erhöht auch langfristig die Wahrscheinlichkeit für einen Schlaganfall. Bei geringem bis moderatem Alkoholkonsum wird der kurzfristige Risikoanstieg möglicherweise durch die diesbezüglich eher günstigen Langzeiteffekte aufgehoben.

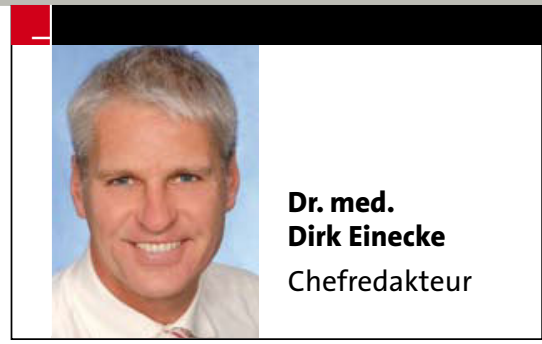

NEUE FORMEL

\title{
Frauenherzen sollten weniger rasen
}

Die traditionelle, auf das männliche Herz bezogene Berechnung der Zielherzfrequenz unter körperlicher Belastung überschätzt die Kapazitäten des weiblichen Pumporgans. Nach einer Studie an 5437 gesunden Frauen im Alter über 35 Jahren liegen die altersbezogenen Trainingsgrenzwerte, die Sportlerinnen ohne Risiko empfohlen werden können, deutlich unter der bisherigen Obergrenze. Die Formel für den Zielwert bei Frauen wurde entsprechend modifiziert. Während man nach der alten Formel das Alter von 220 subtrahiert, lautet die neue, adaptierte Formel: 206 minus $\mathbf{8 8 \%}$ des Alters.

Gulati $M$ et al. Circulation 2010; DOI:10.1161/CIRCULATIOBAHA.110939249

PHARMAKOTHERAPIE BEI KINDERN

Gleich steigt ihr Schlaganfallrisiko.

\section{SELBSTMORDVERSUCH ÜBERLEBT}

\section{Wann die Wiederholungsgefahr groß ist}

Wenn ein Mensch bei einem Suizidversuch mit dem Leben davonkommt, besteht eine nicht geringe Gefahr für eine „erfolgreiche" Wiederholungstat. In einer schwedischen Studie mit fast 50 ooo Personen, die wegen versuchten Selbstmords ins Krankenhaus kamen, nahmen sich $12 \%$ in den darauffolgenden Jahren das Leben. Das Risiko war deutlich erhöht, wenn für den Suizidversuch eine besonders gewaltsame Methode gewählt worden war wie Hängen oder aus großer Höhe Springen. So folgte misslungenen Versuchen, durch Erhängen oder Ertrinken aus dem Leben zu scheiden, sechs- bzw. viermal häufiger eine Selbsttötung als dem (am häufigsten unternommenen) Versuch, sich zu vergiften. Runeson B et al. BMJ, Juli 2010

\section{Auf den Teelöffel ist kein Verlass}

Kinder erhalten Medikamente meist in Saftform. Die Dosis wird dann schon mal mit dem Tee- oder Esslöffel abgemessen. Das kann zu massiven Über- oder Unterdosierungen führen, warnen griechische Wissenschaftler. Besser als haushaltsübliche Löffel sind die geeichten Messlöffel, die in der Regel mitgeliefert werden. Bei kleinen Kindern besteht aber die Gefahr, dass sie sich gegen die Medikamentengabe wehren und einen Teil der Dosis verschütten. Dies ließe sich mit einer geeichten Einmalspritze (natürlich ohne Kanüle) verhindern.

Falagas et al. IJCP 2010;64:1185-1189 